\title{
Magnitude and Factors Contributing to Erectile Dysfunction among Diabetic Men Attending the Diabetic Clinic at Debre Tabor Comprehensive and Specialized, Hospital in North West, Ethiopia 2020, Institutional Based Cross-Sectional Study
}

\author{
Gashaw Mehiret ${ }^{1 *}$, Bizuayehu Dersie ${ }^{2}$, Libsuye Yalgaw $^{3}$ \\ ${ }^{1}$ School of Medicine, College of Health Science, Debre Tabor University, Debre Tabor, Ethiopia \\ ${ }^{2}$ School of Medicine, College of Health Science, Debre Markose University, Debre Markos, Ethiopia \\ ${ }^{3}$ Department of Health Informatics, Debre Tabor College of Health Science, Debre Tabor, Ethiopia \\ Email: ^mehiretg21stdr@gmail.com,dersibuzu@gmail.com, libsuye@gmail.com
}

How to cite this paper: Mehiret, G., Dersie, B. and Yalgaw, L. (2021) Magnitude and Factors Contributing to Erectile Dysfunction among Diabetic Men Attending the Diabetic Clinic at Debre Tabor Comprehensive and Specialized, Hospital in North West, Ethiopia 2020, Institutional Based Cross-Sectional Study. Journal of Diabetes Mellitus, 11, 69-82.

https://doi.org/10.4236/jdm.2021.113006

Received: December 22, 2020

Accepted: June 27, 2021

Published: June 30, 2021

Copyright $\odot 2021$ by author(s) and Scientific Research Publishing Inc. This work is licensed under the Creative Commons Attribution International License (CC BY 4.0).

http://creativecommons.org/licenses/by/4.0/ (c) (i) Open Access

\begin{abstract}
Background: Erectile dysfunction, which is defined as difficult to attain and maintain an erectile function enough to permit sufficient sexual performance, is accepted to be a big problem especially among diabetic patients. Objective To assess the Magnitude and factors contributing to Erectile Dysfunction Among Diabetic men attending the diabetic clinic in Debre Tabor Comprehensive and Specialized hospital, North West Ethiopia. Methods: Hospital based cross-sectional study was conducted on 362 participants in Debre Tabor Comprehensive and Specialized Hospital from August - December 2020 using systematic random sampling technique. Data were analyzed with SPSS Version 23. Binary and multivariable logistic regressions were done to identify factors which were contributing to erectile dysfunction. P-value $<0.05$ and the corresponding 95\% CI of odds ratios were considered to declare the result as statistically significant. Results: Three hundred sixty-two diabetes patients participating in the study with the mean age being $44.4 \pm 14.47$ (range: 18 78 ) years were interviewed. The majority (59.7\% with CI: $54.4: 64.6)$ of the diabetes patients suffered from erectile dysfunction and 13.3\% (95\% CI 17.8\% - 26.8\%) were found to have severe erectile dysfunction. Bi-variable analysis showed duration of diabetes ( $>10$ years), type of diabetes (type II), physical exercise, drinking alcohol, BMI, blood glucose, and blood pressure were associated with erectile dysfunction at $5 \%$ level $(\mathrm{p} \leq 0.05)$. Multiple logistic re-
\end{abstract}


gression analysis revealed that duration of diabetes 10 years $(\mathrm{AOR}=6.2$, 95\% CI: $2.78-13.85, \mathrm{p}=0.001$ ), co-existing hypertension (AOR: 3.59, 95\% CI: $1.58-8.19, \mathrm{p}=0.002)$, physically inactive $(\mathrm{AOR}=2.87,95 \% \mathrm{CI}: 1.53-$ 8.31, $\mathrm{p}=0.003$ ), unsafe level alcohol intake (AOR: 3.09; 95\% CI $1.45-6.59^{\star}, \mathrm{p}$ $=0.003$ ) and raised blood glucose (AOR: 15.26, 95\% CI: $7.82-29.77, \mathrm{p}=$ 0.004 ) were independent risk factors but no association was found with other variables. Conclusion: The magnitude of erectile dysfunction in this study population was $59.7 \%$ and associated with the type of diabetes; duration of diabetic, physical exercise, alcohol drinking, increase in blood pressure, and elevated blood glucose level were independently correlated with erectile dysfunction.

\section{Keywords}

Magnitude Contributing Factors, Erectile Dysfunction, Diabetes Mellitus

\section{Introduction}

Diabetes mellitus is a chronic progressive metabolic disorder characterized by hyperglycemia mainly due to absolute deficiency of insulin hormone (Type 1 $\mathrm{DM}$ ) or relative insulin deficiency or resistance hormone (Type 2 DM). Diabetes mellitus virtually affects every system of the body mainly because of metabolic disturbances caused by hyperglycemia, especially if diabetes control over a period of time proves to be poor [1].

There are lots of causes of ED generally falling into two groups, organic and psychogenic causes. The organic causes are further classified into five categories: vascular, traumatic/post-surgical, neurological, endocrine-induced, and drug-induced. Probably psychogenic causes are depression, anxiety, and relationship problems [2]. Different chronic diseases like diabetes mellitus, cardiovascular disease, and depression are associated with a higher proportion of erectile dysfunction [3] where diabetic mellitus leads to be a major problem [4].

Erectile dysfunction is a common medical complication of diabetes. It is estimated that more than $75 \%$ of diabetic men will be affected at some time in their lives time with a consistent or recurrent inability to achieve and sustain an erection adequate for sexual activity, typically at an earlier age than nondiabetic men [5].

Erectile dysfunction (ED), defined as difficult to attain and sustain an erection sufficient to permit satisfactory sexual performance, is evidenced to be a major problem especially among diabetic patients [1].

While the incidence of ED increases proportionately with age in the general population, the gradient is particularly elevated in diabetic men as they appear to have more severe dysfunction [5]. In the United States alone, more than 53\% of diabetic men are affected by ED [6]. Research suggests that there is a significant relationship between ED and diabetes mellitus (DM) in men and the estimated proportion of $\mathrm{ED}$ in diabetic men has been reported to range from $20 \%$ to $71 \%$ 
[7] [8]. A similar study conducted in Cameron reported that men with DM were more than twice as likely to have ED compared to men without diabetes [9]. Erectile dysfunction can occur early during the disease progression and it can occasionally be the presenting symptom [10]. Some documented reports gave out the main risk factors of ED in people with diabetes as neuropathy, vascular insufficiency, poor glycemic control, hypertension, low testosterone levels, and lifestyle factors such as smoking, alcohol and inactivity. In addition, the prevalence of ED alarmingly increases with age [10] [11].

A wide range prevalence of rates of ED among diabetic men has been reported in various studies in the world. These studies suggested that its prevalence in men with diabetes ranges from $35 \%-90 \%$ versus $26 \%$ in the general population [2] [6] [11] [12] [13] [14].

Erectile dysfunction (impetus) is a big issue in the world particularly in Ethiopia area where health seeking behavior of the population are significantly poor which needs personal privacy; all most all men with the case feel anxious or discomfort to disclose and discuss the problem with their physician, or even if with their life partner. In Ethiopia, open discussions about sexual issues occur rarely we can say has no at all. There is currently a huge gap in the literature, as no adequate studies about ED have previously been conducted in Ethiopia particularly in our region south Gondar Zone Ethiopia. Therefore this study aimed to assess the magnitude and factors which contribute to ED on diabetic patients who attend DTCSH and will contribute to improving the quality of life of diabetic patients including the prevention of unnecessary psychosocial consequences [15].

\section{Methods and Materials}

\subsection{Study Area and Period}

This study was conducted in Debre Tabor Comprehensive and Specialized hospital, with diabetic men attending the diabetic clinic. Debre Tabor town is the capital city of South Gondar Zone, in the Amhara Regional State which a distance of $667 \mathrm{Kms}$ away from Addis Ababa in the North east direction capital city of Ethiopia. There were 857 diabetic cases attended in the hospital as an outpatient follow-up. Diabetes patients were followed at one medical OPD every week on Monday. The study was conducted from August 05 to December $05 / 2020 / 21$.

Hospital-based cross-sectional study design was conducted in Debre Tabor Comprehensive and Specialized hospital. Source population was all adult $(\geq 18$ years) diabetic men clients attending the diabetic clinic in Debre Tabor Comprehensive and Specialized hospital specialized hospital.

\subsection{Study Population}

The study population was all adult ( $\geq 18$ years) patients who have an appointment during the data collection period. 


\subsection{Sample Size Determination and Sampling Technique}

\subsubsection{Sample Size Determination}

The sample size was determined using a single population proportion formula. The proportion of erectile dysfunction was considered $69 \%$ since previous studies were conducted in hospitals of central and North-western zone of Tigray, northern Ethiopia, 2017. Then, the following assumptions were used: level of confidence was $95 \%$ (1.96), 5\% marginal error. Based on these assumptions, the sample size was calculated. After the 10\% nonresponsive rate considered, $329 \%$ $+10 \%$, the total sample size for the study was 362 .

$$
\begin{aligned}
n & =\frac{Z_{1-\alpha / 2}^{2}(p)(1-p)}{d^{2}} \\
& =\frac{(1.96)^{2}(0.69 \times 0.31)}{(0.05)^{2}} \\
& =329
\end{aligned}
$$

where:

$Z_{1-\alpha / 2}^{2}=$ critical value at $95 \%$ confidence level of certainty (1.96).

$P=$ proportion of erectile dysfunction.

$d=$ the margin error between the sample and the population.

After $10 \%$ nonresponsive rate considered, $329 \%+10 \%$ the total sample size for the study was 362 .

\subsection{Sampling Technique}

Samples were selected from 857 diabetic patients who were registered and have regular follow-up at follow-up clinic. Most of the patients were appointed during the interval of four to six weeks. However, for some patients, the frequency of their visits can vary depending on their blood glucose level. Then the total number of diabetic patients who had follow-up from August, 5/2020 to December 05/2020 was taken to estimate the current patient flow. Then a systematic sampling technique was employed to approach the study participants. Then we determine the k-value by dividing the total number of patients by the sample size, i.e., $857 / 362=2.36 \approx 2$. Then the first patient was selected by lottery method from the first two patients. Then the study participant was selected every two patients until the required sample size was achieved.

All diabetic men clients attending the diabetic clinic and able to give informed consent were eligible for this study. Patients who were eligible for participation included men with type 1 and type 2 diabetes receiving pharmacological treatment for the disease.

\subsection{Operational Definitions/Operation of the Term}

The IIEF-5-point scale would be used to determine the presence and severity of ED with a possible score totaling 5 to 25 .

ED: Study participants who scored $\leq 21$ out of 25 points [16].

Severe ED: Study participants who scored $1-7$ out of 25 points. 
Moderate ED: Study participants who scored 8 - 16 out of 25 points.

Mild ED: Study participants who scored 17 - 21 out of 25 points [16].

Adequate glycemic control for DM: FBS measurement $70 \mathrm{mg} / \mathrm{dL}-126 \mathrm{mg} / \mathrm{dL}$ [5].

Patients with diabetes are defined as anyone using insulin, oral hypoglycemic medication, or a combination of both.

Hypertension was defined as SBP $\geq 130 \mathrm{mmHg}$ and/or DBP $>80 \mathrm{mmHg}$ or known hypertensive on treatment.

\subsection{Data Collection Instruments}

Information regarding erectile function was collected through a structured questionnaire which included the abridged five-item version of the International Index of Erectile Function (IIEF-5) [12]. The IIEF questionnaire assesses and measures five components: erection ability, orgasmic (ejaculation) function, sexual desire (libido), intercourse pleasure (ability to maintain intercourse), and overall satisfaction/premature ejaculation domains. It classifies individual sexual function domains into mild, moderate, or severe forms depending on the scores', the specific scores of the mild, moderate, or severe forms were advised to be provided here for better understanding. Additional parameters assessed included; age, BMI, marital status, alcohol consumption, type of diabetic, diabetes related complications, other medical history, and current use of medications. When necessary, the information given by the participants was confirmed with their medical records.

The data from participants were collected using an interview-administered questionnaire and document review. The questionnaire was originally developed in English and then translated into Amharic (Ethiopian National language) and then back to English by a language expert. The Amharic language questionnaire was used to collect data. The wording and sequence of questions was designed in such a way that the logical flow of ideas (from general to specific, from impersonal to personal, and from easy to difficult questions) was maintained. Data was collected by two trained data collectors (clinical nurse) in separate rooms immediately after completing their follow-up.

To assure quality of the data, the following measures were undertaken: The questionnaire validity was assessed by public health, reproductive health, clinicians and psychiatrists in hospitals, before the actual data collection would occur and unnecessary instruments (tools) avoid. Intensive training was given to all supervisors and data collectors. Overall activity was controlled by the principal investigator, who was supervised carefully during data collection.

The data was checked for completeness and consistency. Data cleaning was made manually by removing missing ideas and responses to questions about relevant information. Then the data was checked for completeness, coded, entered into EPI data 4.1 (define data, make data file, cheek, enter document), and transported to SPSS version 23.00 statistical software for analysis. Computer 
frequencies were used to check for missed variables. Any errors were identified at this time and then would be retrieved using the code numbers. Descriptive statistics were used to characterize the variables. Binary logistic regression was done with a statistical significance of $p \leq 0.05$ was used to determine the relationship between risk factors and social demographics. Additionally; the odds of patient susceptibility to ED was examined using the odds ratio calculation at $95 \%$ confidence level. In many instances, recoding of the variables into categories was done to achieve an optimal statistical test.

Ethical clearance and approval was obtained from the ethical review Board of Debre Tabor University Health Science College. Letter from Debre Tabor University, College of Health Science, was submitted to hospital administrators to obtain their co-operation. All study participants were informed about the purpose of the study and any additional information was given as they need, verbally and in written form.

\section{Result}

\subsection{Socio-Demographic Characteristics of the Study Population}

This study shows that three hundred sixty-two (362) patients were interviewed to achieve the study with a $100 \%$ response rate. The mean age of the study participants was $44.4 \pm 14.47$ years (range: 18 - 78 years). The mean duration of a DM diagnosis was $6.22 \pm 1.02$ years (range: 2 months - 25 years). Almost all 312 (86.2\%) of the participants were of Amhara ethnicity, 47 (11.9\%) were as the rest of them were other ethnic groups, and regarding marital statues the majority (52.8\%) were also married. Participants were also predominantly of the Orthodox Christian religion followers (76.8\%) and 110 (30.4\%) were unable to read and write and $51(14.1 \%)$ were high school and above regarding educational status. Concerning occupational status most participants were governmentally employed (72.9\%) with monthly income, and most of them were below 3000 birr (89.6\%) (Table 1).

\subsection{Clinical and Life Style Characteristics of Participants}

The participant's life style factors demonstrated that 88 (24.3\%) drunk alcohol, 21 (2.8\%) chewing chat, 284 (78.5\%) of them had no regular Physical exercise and, $275(75.9 \%)$ of the study participants their BMI were in the normal range, only 78 (21.5\%) of them were Overweight. Clinical characteristics of the participants 206 (56.9\%) were type 1 of the total DM participants, 138 (38.1\%) were $\geq 10$ years duration of DM, and regarding blood glucose level indicated that FBS levels of study participants, 164 (45.3\%) of the participants were in the normal range $(<126 \mathrm{mg} / \mathrm{dl})$ and $198(54.7 \%)$ of participants were above the normal range $(\geq 126 \mathrm{mg} / \mathrm{dl})$ which showed that the majority of participant's had poor glycemic control status at the time of the study was conducted. Sixty-two participants (17.2\%) had additional chronic disease beside DM. The most common chronic disease co-existing with DM was hypertension, which accounted for 
Table 1. Socio-demographic characteristics of diabetic men attending the diabetic clinic at Debre Tabor Comprehensive and Specialized Hospital, South Gondar zone, North West Ethiopia 2020/21 ( $=362)$.

\begin{tabular}{ccc}
\hline Variable & Category & Number (\%) \\
\hline \multirow{2}{*}{ Age in years } & $<30$ & $81(22.4)$ \\
& $30-44$ & $104(28.7)$ \\
& $45-59$ & $100(27.6)$ \\
& $\geq 60$ & $77(21.3)$ \\
\hline Marital status & Single & $61(16.9)$ \\
& Married & $191(52.9)$ \\
& Divorce & $77(21.3)$ \\
Religion & Widowed & $33(9.1)$ \\
& Orthodox & $278(76.8)$ \\
& Muslim & $73(20.2)$ \\
& Protestant & $11(3)$ \\
\hline Educational level & Cannot read and write & $110(30.4)$ \\
& Can read and write & $94(26)$ \\
& Primary school & $35(9.7)$ \\
& High school & $51(14.1)$ \\
& Collage \& university & $72(19.9)$ \\
\hline
\end{tabular}

$21 \%$ of cases. In DM patients, only $2.8 \%$ were used erection medication (Table 2).

\subsection{Magnitude of Erectile Dysfunction}

The magnitude of ED was found to be 216 (59.7\%) with (CI: 54.4:64.6) and the mean score on the International Erectile Function Test was 17.1602 \pm 7.3372 (range: 5 - 25) of which 13.3\% had severe ED, 35.1\% had moderate ED, and 11.3\% had mild ED. Only $30.1 \%$ of patients had normal erectile function (Figure 1).

\subsection{Severity of Erectile Dysfunction}

As in the graph below; showed that the magnitude of erectile dysfunction and its severity was increased with age, the respondents whose age $\geq 60$ years old, 26 (7.18\%) were severe, 47 (13\%) were moderate and $2(0.55 \%)$ were mild complications, at the young age of the respondents the complication almost negligible (Figure 2).

\subsection{Factors Contributing with Erectile Dysfunction}

On binary logistic regression analysis, there was an association between ED and type of diabetic, duration of diabetes, physical exercise, drinking alcohol, BMI, blood glucose, and blood pressure. Whereas in multivariate logistic regression 
analyses showed that there was an association between ED and type of diabetic, duration of diabetes, physical exercise, drinking alcohol, blood glucose, and blood pressure. Respondents who had a diagnosis of type two DM were 1.95

Table 2. Clinical and life style characteristics of diabetic men attending the diabetic clinic at Debre Tabor Comprehensive and Specialized Hospital, South Gondar zone, North West, Ethiopia, 2020/21 ( $=362)$.

\begin{tabular}{|c|c|c|}
\hline Variable & Category & Number (\%) \\
\hline \multirow{2}{*}{ Type of DM } & Type 1 & $206(56.9)$ \\
\hline & Type 2 & $156(43.1)$ \\
\hline \multirow{2}{*}{ Duration of DM } & $<10$ years & $224(61.9)$ \\
\hline & $\geq 10$ years & $138(38.1)$ \\
\hline \multirow{3}{*}{ Medication used } & Oral hypoglycemic & $155(42.8)$ \\
\hline & Insulin & $20356.1(56.1)$ \\
\hline & Both & $1(0.3)$ \\
\hline \multirow{2}{*}{ Physical exercise } & Yes & $78(21.5)$ \\
\hline & No & $284(78.5)$ \\
\hline \multirow{2}{*}{ Time of physical exercise per week } & $<3$ times & $3(0.8)$ \\
\hline & $\geq 3$ times & $75(20.7)$ \\
\hline \multirow{3}{*}{ BMI } & Under weight & $9(0.025)$ \\
\hline & Normal & $275(75.9)$ \\
\hline & Over weight & $78(21.5)$ \\
\hline \multirow{2}{*}{ Blood glucose level } & $<126 \mathrm{gm} / \mathrm{dl}$ & $164(45.3)$ \\
\hline & $\geq 126 \mathrm{gm} / \mathrm{dl}$ & $198(54.7)$ \\
\hline \multirow{2}{*}{ Diabetics complication } & Yes & $138(38.1)$ \\
\hline & No & $224(61.9)$ \\
\hline
\end{tabular}

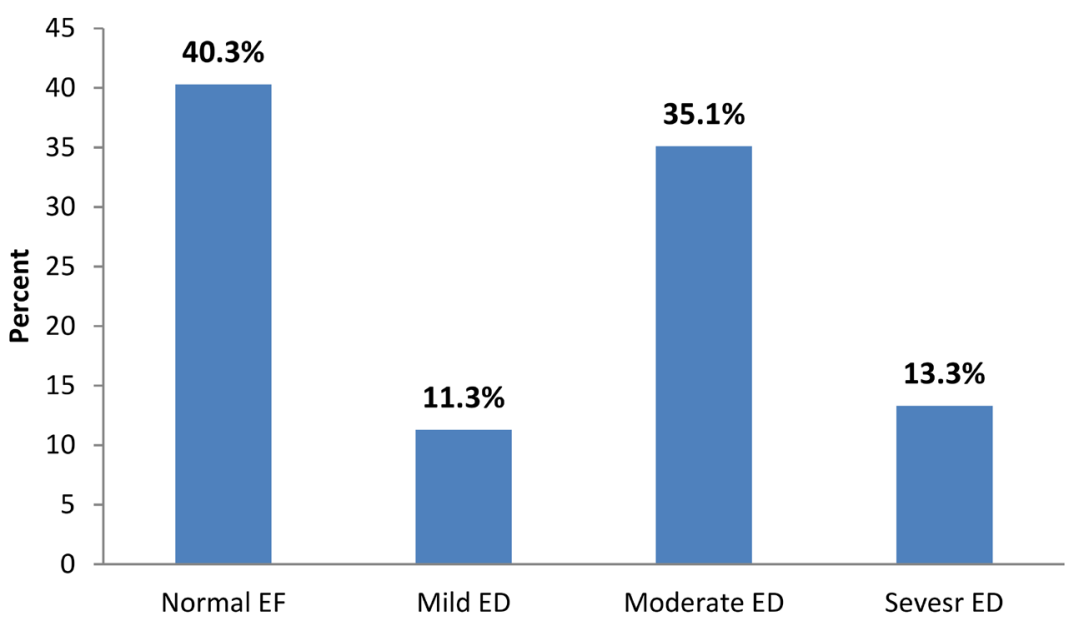

Figure 1. Distribution of severity of erectile dysfunction among diabetics attending at Debre Tabor Comprehensive and Specialized Hospital, South Gondar zone, North West, Ethiopia, 2020/21 (362). 


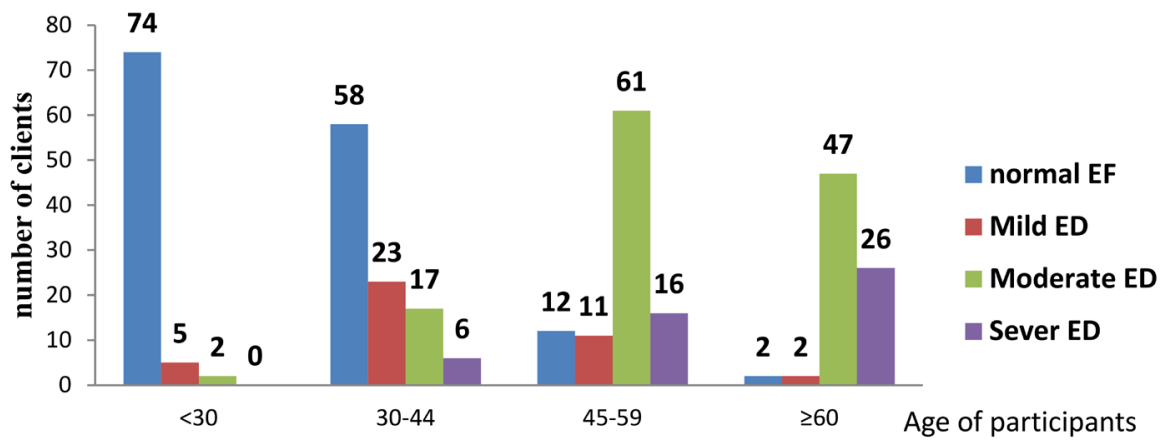

Figure 2. Severity of erectile dysfunction by age group among male Diabetic clients attending Diabetic Clinic at Debre Tabor Comprehensive and Specialized Hospital, South Gondar zone, North West Ethiopia, 2020/21.

times more likely to have ED than type one DM (AOR 1.95, 95\% CI: 1.007 - 3.76, $\mathrm{p}=0.048)$. On the other, hand respondents who had a diagnosis of DM for more than 10 years were 6.2 times more likely to have ED compared to those who had a diagnosis of diabetes for less than 10 years with (AOR $=6.2,95 \% \mathrm{CI}: 2.78$ $13.85, \mathrm{p}=0.001$ ). And, study participants who were physically inactive were 3.57 times more likely to have ED compared to those who did physical exercise regularly $(\mathrm{AOR}=2.87,95 \% \mathrm{CI}: 1.53-8.31, \mathrm{p}=0.003)$ and those who had alcohol drinking 3 times the probability to develop ED than not drinking. As blood pressure increases, the odds of having ED increased significantly, men with hypertensive were almost 3.59 times more likely to develop ED than those with normal blood pressure (AOR: $3.59,95 \%$ CI: $1.58-8.19, \mathrm{p}=0.002$ ) and as blood glucose increase odds of having ED than normal blood glucose level which were 15.26 times more likely to develop ED with (AOR: 15.26, 95\% CI: $7.82-29.77, \mathrm{p}=0.004)$. Otherwise, no associations were found between other variables (Table 3 ).

\section{Discussion}

In our study, the majority (50.9\%) of patients were Type 1 DM than Type 2 DM. It further confirms that type of diabetic, duration of DM, physical exercise, alcohol drinking, increase in blood pressure, and blood glucose level were independently correlated with the ED.

The magnitude of erectile dysfunction in all stages was $59.7 \%$ among men diabetic clients aged $\geq 18$ years, which is comparable with several studies, which have been conducted in Egypt, Nigeria, Jordanian and, Jamaica (63.6\%), (57.4\%), (62\%), and (64\%) respectively [14] [16] [17] [18]. However, less than the study conducted in north-western zone of Tigray, there were $69.9 \%$, this may be due to sample size difference and time elapsed and in Pakistan $80.8 \%$ may be different population cultures, Socio-demographic risk factors and methodology. In this study, type two diabetic had strong associations with ED which were also found in a study conducted in the United Arab Emirates, because this may be the most type two diabetic presented with complications of the disease. 
Table 3. logistic regression of factors affecting erectile dysfunction of diabetic patients in Debre Tabor Comprehensive and Specialized Hospital, South Gondar zone, North West Ethiopia, 2020/21 ( $\mathrm{N}=362)$.

\begin{tabular}{|c|c|c|c|c|c|}
\hline \multirow{2}{*}{ Variables } & \multicolumn{2}{|c|}{ Erectile dysfunction } & \multirow{2}{*}{ COR (CI: 95\%) } & \multirow{2}{*}{$\operatorname{AOR}(95 \% \mathrm{CI})$} & \multirow{2}{*}{ P-value } \\
\hline & No & Yes & & & \\
\hline \multicolumn{6}{|l|}{ Type of DM } \\
\hline Type 1 & 101 & 105 & 1 & 1 & \multirow{2}{*}{0.048} \\
\hline Type 2 & 45 & 111 & $2.37(1.53-3.69)$ & $1.95(1.007-3.76)^{\star}$ & \\
\hline \multicolumn{6}{|l|}{ Duration of DM } \\
\hline$<10$ years & 133 & 91 & 1 & 1 & \multirow{2}{*}{0.001} \\
\hline$\geq 10$ years & 13 & 125 & $14.05(7.48-26.39)$ & $6.20(2.78-13.85)^{\star}$ & \\
\hline \multicolumn{6}{|l|}{ Alcohol drinking } \\
\hline Yes & 23 & 65 & $2.30(1.35-3.92)$ & $3.09(1.45-6.59)^{\star}$ & \multirow{2}{*}{0.003} \\
\hline No & 123 & 151 & 1 & 1 & \\
\hline \multicolumn{6}{|l|}{ Physical exercise } \\
\hline Yes & 54 & 24 & 1 & 1 & \multirow{2}{*}{0.003} \\
\hline No & 92 & 192 & $4.69(2.73-8.07)$ & $3.57(1.53-8.19)^{*}$ & \\
\hline \multicolumn{6}{|l|}{ BMI } \\
\hline Over weight & 9 & 75 & $8.1(3.9-16.81)$ & $1.16(0.401-3.35)$ & \multirow{2}{*}{0.78} \\
\hline Normal & 137 & 141 & 1 & 1 & \\
\hline \multicolumn{6}{|l|}{ Blood pressure } \\
\hline Hypertensive & 12 & 88 & $7.68(4.01-14.71)$ & $3.59(1.58-8.19)^{*}$ & \multirow{2}{*}{0.002} \\
\hline Normal & 134 & 128 & 1 & 1 & \\
\hline \multicolumn{6}{|c|}{ Blood sugar level } \\
\hline$\geq 126 \mathrm{gm} / \mathrm{dl}$ & 23 & 175 & $22.82(13.0339 .97)$ & $15.26(7.82-29.77)^{\star}$ & \multirow{2}{*}{0.004} \\
\hline$<126 \mathrm{gm} / \mathrm{dl}$ & 123 & 41 & 1 & 1 & \\
\hline
\end{tabular}

-COR (95\% CI): crude odds ratio with 95\% confidence interval; -AOR (95\% CI): adjusted odds ration with $95 \%$ confidence interval; ${ }^{*}$-significantly associated category variables at $\mathrm{p}<0.05$.

The duration of diabetes and the magnitude and degree of ED had a strong relationship, longer duration of DM could be associated with poor glycemic control, and its connection with ED has been well documented by several trials. Consistent with previous studies, a positive association between the duration of DM and the magnitude of ED was found in the present study, which showed that as the duration of diabetes increases, the odds of having ED increased significantly, where men with a history of DM $>10$ years were six times more likely to report ED than those with a history of $<10$ years (AOR: 6.20, 95\% CI: 2.78 $13.85, \mathrm{p}=0.001)$. Further analysis showed that the severity of ED increased with the duration of DM. The research data were in accordance to north-west Tigray and Saudi patients where men with a history of $\mathrm{DM}>10$ years were four and three times more likely to report ED than those with a history of $<10$ years re- 
spectively [15] [19]. This association noted above might be attributed to the natural progression of the disease and the interrelated complications, in particular vascular disease, that are more likely to develop over time. Nevertheless, there are several factors that may confound the relationship between the duration of $\mathrm{DM}$ and ED, such as the presence of comorbid conditions (e.g., hypertension which is significantly associated). On the other hand, alcohol drinking and physical activity were found to correlate significantly $(\mathrm{p}<0.05)$ but there was no association with other variables which had been associated in other study.

The study also further suggests that monthly income, sedentary life (poor physical activity), drinking alcohol, poor controlled blood glucose, and blood pressure are independent predictors for ED and its severity. In contrast, this study reveals the age of the participants, BMI, co-morbidity, taking concomitant drugs are not associated with ED.

\section{Conclusion}

The results of this study have indicated that ED is highly prevalent among study area men with diabetes. The frequency as well as the severity of ED increased with age in patients with diabetes and had significant correlation with the type of diabetic, duration of diabetes, physical activity, alcohol drinking, increase in blood pressure and blood glucose. Assessment and management of ED should be addressed and part of routine medical care in diabetic follow-up clinics. Health-care providers should openly ask men with chronic disease, particularly those with diabetes, about symptoms of ED. Clients who are of older age and who have a longer duration of DM, physically inactive, and those who take alcohol require special attention in screening for ED.

\section{Limitations}

Since this study has used cross-sectional design, we cannot report the cause and effect. Future studies should examine the causal relationship of the variables using analytical study design. The issue of social desirability bias should also be considered in the current study. A detailed evaluation of the long-term glycemic control, lipid profile, and smoking could improve the validity of the study.

\section{Authors' Contributions}

GM designed the study, collected and analyzed the data, interpreted the findings, and wrote the manuscript. $\mathrm{BD}$, and LY were involved in the design, data analysis and critically revised the manuscript. All authors read and approved the final manuscript.

\section{Acknowledgements}

The authors are grateful to Debre-Tabor University for giving ethical approval to conduct this research. We sincerely thank the study participants for their time to participate in this study. 


\section{Availability of Data and Materials}

The datasets used and analyzed during the current study are available from the corresponding author on reasonable request.

\section{Consent for Publication}

Not applicable.

\section{Ethical Approval and Consent to Participate}

The study protocol was approved by Ethical Clearance Board of College of Medicine and Health Sciences of Debre Tabor University. Before any attempt at interview, written informed consent was obtained from each respondent. Their participation was voluntary and they were informed about their right to withdraw from responding whenever they like and not to respond to the questions they want or not to obtain in due course were confidentially stored. Those study individuals, who suffered from DM complications, especially ED, were advised to disclose the problem to their physicians since the problem might be solved either by pharmacologic or other psychological therapy.

\section{Funding}

This study is a self-funded research study.

\section{Conflicts of Interest}

The authors declare no conflicts of interest regarding the publication of this paper.

\section{References}

[1] NIH Consensus Conference (1993) Impotence: NIH Consensus Development Panel on Impotence. JAMA, 270, 83-90. https://doi.org/10.1001/jama.1993.03510010089036

[2] Chu, N.V. and Edelman, S.V. (2001) Diabetes and Erectile Dysfunction. Clinical Diabetes, 19, 45-47. https://doi.org/10.2337/diaclin.19.1.45

[3] Kubin, M., Wagner, G. and Fugl-Meyer, A.R. (2003) Epidemiology of Erectile Dysfunction. International Journal of Impotence Research, 15, 63-71. https://doi.org/10.1038/sj.ijir.3900949

[4] Shiri, R., Hakama, M., Häkkinen, J., Tammela, T.L.J., et al. (2005) Relationship between Smoking and Erectile Dysfunction. International Journal of Impotence Research, 17, 164-169. https://doi.org/10.1038/sj.ijir.3901280

[5] de Tejada, I.S., Anglin, G., Knight, J.R. and Emmick, J.T. (2002) Effects of Tadalafil on Erectile Dysfunction in Men with Diabetes. Diabetes Care, 25, 2159-2164. https://doi.org/10.2337/diacare.25.12.2159

[6] Selvin, E., Burnett, A.L. and Platz, E.A. (2007) Prevalence and risk factors for erectile dysfunction in the US. The American Journal of Medicine, 120, 151-157. https://doi.org/10.1016/j.amjmed.2006.06.010

[7] Cho, N.H., Ahn, C.W., Park, J.Y., Ahn, T.Y., Lee, H.W., Park, T.S., et al. (2005) 
Prevalence of Erectile Dysfunction in Korean Men with Type 2 Diabetes Mellitus. Diabetic Medicine, 23, 198-203. https://doi.org/10.1111/j.1464-5491.2005.01789.x

[8] Siu, S.C., Lo, S.K., Wong, K.W., Ip, K.M. and Wong, Y.S. (2001) Prevalence of and Risk Factors for Erectile Dysfunction in Hong Kong Diabetic Patients. Diabetic Medicine, 18, 732-738. https://doi.org/10.1046/j.0742-3071.2001.00557.x

[9] Sun, P., Cameron, A., Seftel, A., Shabsigh, R., Niederberger, C. and Guay, A. (2006) Erectile Dysfunction-An Observable Marker of Diabetes Mellitus? A Large National Epidemiological Study. The Journal of Urology, 176, 1081-1085. https://doi.org/10.1016/j.juro.2006.04.082

[10] Braun, M., Wassmer, G., Klotz, T., Reifennah, B., Mathers, M. and Engelman, U. (2000) Epidemiol of ED: Results of the Cologne Male Survey. International Journal of Impotence Research, 12, 305-311. https://doi.org/10.1038/sj.ijir.3900622

[11] Marumo, K., Nakashima, J. and Murai, M. (2001) Age-Related Prevalence of Erectile Dysfunction in Japan: Assessment by the International Index of Erectile Function. International Journal of Urology, 8, 53-55.

https://doi.org/10.1046/j.1442-2042.2001.00258.x

[12] Feldman, H.A., Goldstein, I., Hatzichristou, D.G., Krane, R.J. and Mckinlay, J.B. (1994) Impotence and Its Medical and Psychosocial Correlates: Results of the Massachusetts Male Aging Study. Journal of Urology, 151, 54-61. https://doi.org/10.1016/S0022-5347(17)34871-1

[13] United States Bureau of Census (1992) Statistical Abstract of the United States 1992. 112th Edition, Washington DC, 19.

[14] Shaeer, K.Z., Osegbe, D.N., Siddiqui, S.H., Razzaque, A., Glasser, D.B. and Jaguste, V. (2003) Prevalence of Erectile Dysfunction and Its Correlates among Men Attending Primary Care Clinics in Three Countries, Pakistan, Egypt, and Nigeria. International Journal of Impotence Research, 1, S8-S14. https://doi.org/10.1038/sj.ijir.3900971

[15] Seide, A., et al. (2017) Prevalence and Determinants of Erectile Dysfunction among Diabetic Patients Attending in Hospitals of Central and Northwestern Zone of Tigray, Northern Ethiopia: A Cross-Sectional Study. BMC Endocrine Disorders, 17, 16. https://doi.org/10.1186/s12902-017-0167-5

[16] Al-Hunayan, A, Al-Mutar, M., Kehinde, E.O., Thalib, L. and Al-Ghorory, M. (2007) The Prevalence and Predictors of Erectile Dysfunction in Men with Newly Diagnosed with Type 2 Diabetes Mellitus. BJU International, 99, 130-134. https://doi.org/10.1111/j.1464-410X.2006.06550.x

[17] Khatib, F.A., Jarrah, N.S., Shegem, N.S., Bateiha, A.M., Abu-Ali, R.M. and Ajlouni, K.M. (2006) Sexual Dysfunction among Jordanian Men with Diabetes. Saudi Medical Journal, 27, 351-356.

[18] Peter, J., Riley, C.K., Layne, B., Miller, K. and Walker, L. (2012) Prevalence and Risk Factors Associated with Erectile Dysfunction in Diabetic Men Attending Clinics in Kingston, Jamaica. Journal of Diabetology, 2, 2.

[19] Fedele, D., Bortolotti, A., Coscelli, C., Santeusanio, F., Chatenoud, L., Colli, E., et al. (2000) Erectile Dysfunction in Type 1 and Type 2 Diabetics in Italy. International Journal of Epidemiology, 29, 524-531. https://doi.org/10.1093/ije/29.3.524 


\section{Acronyms/Abbreviations}

BMI: Body Mass Index, CAD: Coronary Artery Disease, DBP: Diastolic Blood Pressure, DTCSH: Debre Tabor Comprehensive and Specialized Hospital, DM: Diabetes Mellitus, ED: Erectile Dysfunction, IIEF: International Index of Erectile Function, SBP: Systolic Blood Pressure, SPSS: Statistical Package for Social Science 